\title{
1. Introduction to operations strategy
}

\subsection{WHAT IS OPERATIONS AND WHY IS IT IMPORTANT?}

The operations element of a business is effectively the part of the business that transforms the input into an organisation into the output that gives additional value to the end user than the sum of the inputs to the system. Drucker (1955) stated that it was not the ability to carry a more difficult activity or an activity more efficiently than a competitor that allowed operations to compete within the market place, but rather the ability to provide value for the customer. By appreciating this and continuing to develop the value an organisation has added to the inputs in line with the requirements of the market, the organisation should be able to remain competitive without the need to compete on price. What this means in regard to operations, is that it is the processes that take place within the operations function that allow an organisation to be present and compete within a market place. This is not to say that other functions within an organisation are any less important than operations; however, if an organisation is not carrying out or at least coordinating processes that meet these requirements, the organisation is effectively not contributing by adding value to society.

By focusing on the development of operations within an organisation it becomes possible for the organisation to effectively develop and define the value it creates for the end user to meet their needs in a way that other organisations are unable to do. Rather than focusing on the business functions that support operations, the developments translate directly to how the end user perceives the organisation by the elements that will ultimately relate to their satisfaction. Organisations that have focused on the development of the operations are the organisations that have been able to show long-term growth and success within the market place. Importantly, in these situations, success has not been dictated by a particular innovation, although innovation is important; instead it is their ability to continue to satisfy their customer that defines their performance. By focusing on operations, Toyota has been able to remain profitable where other firms have struggled and it has not had to offer products that redefine the market place. It is able to offer consistently satisfactory products that 
continue to change to match the needs of the market in a manner that uses resources in an efficient manner.

The operational element may be thought of as quite an aggregated element of analysis and difficult to focus specific improvements on, especially if a large range of products and processes are being carried out within an operations function. From a different lower-level perspective, individual processes could be thought of as too disaggregated to be able to develop in a way that will affect over-all performance. For this reason, a useful unit of analysis within the operations functions is that of capabilities, which are able to define how well an organisation is able to compete. A capability is a selection of processes and skills that can be effectively combined in a manner that directly contributes to the value the end user receives. Within the operations functions it is the coordinated development and deployment of capabilities that will be what determines if an operations function is able to perform in a manner that allows an organisation to compete. Particular capabilities that may give organisations competitive advantages may be elements that enable a number of business functions to focus their activities in a way that will be appreciated by the end user (Barney 1991).

\subsection{THE CONCEPT OF STRATEGY}

Before a suitable introduction to operations strategy is possible, it is first necessary to understand why there is a need for an operations strategy. Even before this, it is also necessary to understand why strategy is such a significant concept in business that warrants the amount of interest that it generates. For many years it has been considered necessary to be able to drive a company so that it is able to grow and continue making money for those that have money invested in the company. Unfortunately, this puts unnecessary focus on the results of business rather than focusing on the business means - can a business's success be gauged wholly on something that is simply a product of such a large number of elements? For this reason, it seems self-perpetuating for a company to review its own financial data to determine how it should operate in the future.

The definition of strategy seems to be heavily focused on its connotations in planning, but the subtle difference is that the planning is specifically long range in nature. Before discussing strategy in a business sense, the importance and effect of strategy will be discussed in other areas where long-range planning needs careful consideration. Sport and warfare are both examples of where suitable long-range planning can have significant effects on an outcome, and where focusing on the result alone may not be a suitable way to determine performance. With traditional, purer sports, 
the effects of natural ability are notable, with certain nations being more proficient at certain sports, where genes may assist in certain activities. However, due to people's almost infinite capacity to learn, the cognitive element becomes considerably more important. For athletes, the need to train could be considered a strategy to improve performance and build on natural ability, allowing a gradual improvement in performance over time. With the purer sports, although nature undoubtedly plays a big role in the performance of an athlete, techniques can allow step changes in the performance, with particularly successful ones being passed from one athlete to the next (e.g. Dick Fosbury).

As the complexity of activities increases, the importance of other aspects of ability becomes apparent: all too often athletes fail to perform at big events, even if their ability and technique put them on top on paper. In this case, it is another cognitive element that becomes important: the ability to use the correct ability and technique when required. To reflect this aspect, the use of sports psychologists has become more widespread to give athletes a mental edge, even in the purer disciplines. Performing well on a practice court or in a semi-finals will not allow someone to win - they have to be able to continue to perform in all situations. This aspect of sport seems to become even more significant when considered in regards to more complex sports, when deficiencies in physical, technical or mental ability will all affect overall performance. This is effectively another type of strategy that is employed to combine with the other types, where the athlete's ability in one area will neither determine success nor shape the following year's training plan.

When these ideas are developed and directed onto team sports, the mental elements are even more noticeable, especially in certain sports that rely on certain set plays. However, in other, less structured sports, developing such a capability is considerably more complex, as it requires the team as a whole to be able to adapt in an effective way without explicit direction from a coach or manager. This could be considered a team culture or dynamic where the importance of individual ability, technique and mental strength is second to how well the team functions as a whole. Here, it may be the presence of a managerial figure, who is able to create, develop or destroy this team element. A consistent element within the team could also potentially be as important as team make-up, where structure and culture are created around a particular element that may affect the team performance more than having a star player who does not complement elements already present within the team.

The final description of strategy in a non-business context is easily the earliest identified study, with writing from as early as 2000 BC. Although strategy in such a context is obviously very different from the above 
instances, there are similarities with the extinction of a species (within nature) or loss in a sports event effectively being another form of defeat. However, with the consequences of war being considerably more serious, the associated studies of past successful military strategies began much earlier than such thinking in the sports world. It has long been considered that effective military strategies are what are required for victory, but like the sporting analogy, focusing on a single component will seldom result in the required levels of performance. Such examples were demonstrated within the American civil war, where generals employed Napoleonic strategies without considering the effects of innovations such as long-range rifles on the situations.

Such an example is one of many where those in charge do not take account of how the situations have changed since the campaigns they studied took place. Military strategy could be considered in the same manner as the cognitive elements described above - that although they assist in overall performance they should not be focused upon to the exclusion of other information. An interesting example is Hannibal's defeat to the Romans, who were aware Hannibal's forces were considerably more able, but by employing a strategy of numerous controlled battles, they were able to consistently wear down morale. Even though a number of battles were lost, the overall aim of victory was achieved by understanding the importance of specific elements required for good performance. Although ability, technology, technique and morale are important in performance, through focusing on a specific element, the reduction in overall performance can be sufficient. Having said this, it may simply be specific abilities present in one's force compared to another that determine victory, or at least make defeat less likely.

In the military context, specific analogies to business become even more noticeable, where competing organisations do battle in the market place. The following sections will aim to outline how businesses approach strategy in different ways. With the consideration of this first section, the reason for its inclusion will be apparent, as although the fields and subject matter could not be more different, the common theme of strategy is relatively consistent throughout. This look at strategy also tries to outline the comment earlier that a single measurement, especially one that is a product of so many factors as profit, cannot be a suitable measure of performance. Would measuring a sports team's ability on a single performance be an appropriate way to prepare for the following year, or an army's future performance based on a single victory? Without taking account of many factors, it is simply not possible to gauge performance or prepare for the future. The next section describes how this is done when the primary element of information for the strategy process is financial data. 


\subsection{HISTORIC APPROACHES TO STRATEGY}

In a traditional hierarchically structured company, the way strategy is approached in its most general way is by the collections of large amounts of business information for processing by the most intelligent people in the company. Situated within a headquarters, these highly intelligent people are charged with analysing the company data to determine the most appropriate course of action over the next planning period. Due to the processes required in large businesses, it is likely that the majority of this information will be financial but it is also likely that there will be some market-related data. The result of analysing this data will be an outline of how the company should look in the future, including what acquisitions and liquidations are necessary. Although assessing performance in this way, using financial data as the base unit of analysis, is inappropriate, it does allow the business to be assessed in a systematic way, using wellgrounded Newtonian-based, economic principles.

The data and subsequent strategic plan, although fundamentally flawed, even if the financial data is accurate and unbiased, are considered a good foundation for this activity, as it means that the strategic process can be removed from actual business activities. This potentially gives investors confidence that if those in charge of the direction of the company are skilled at managing money, they should be able to make money for them. This creates a vicious circle, as shown in Figure 1.1, where those able to carry out this function require a grounding in finance rather than the

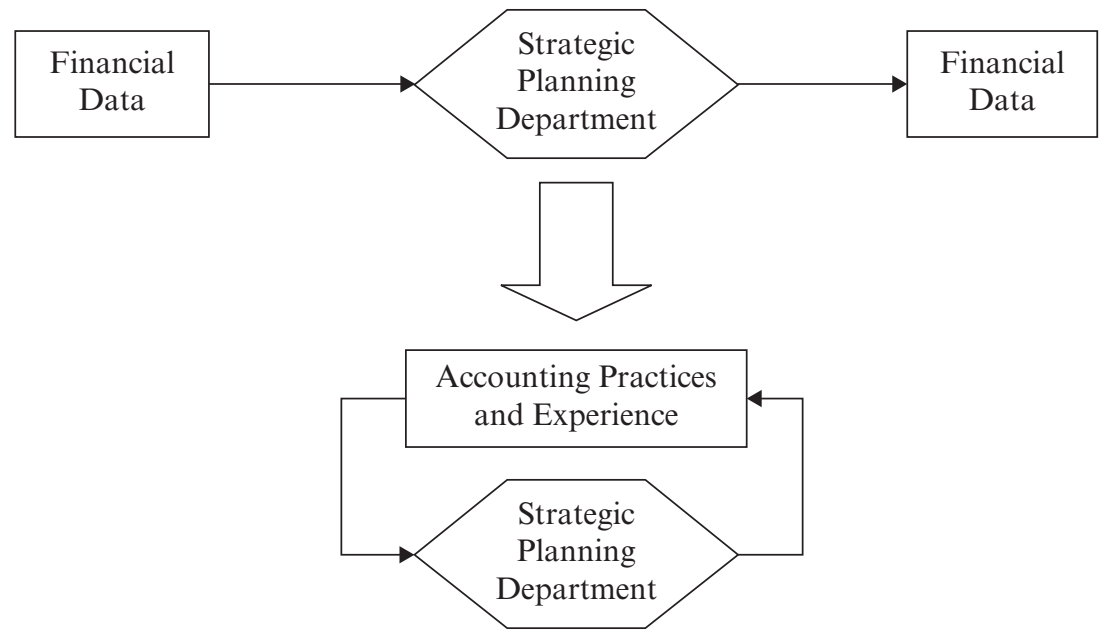

Figure 1.1 Purely financially driven development process 
actual business function. However, is it possible to consider that a business makes money, simply because it makes money, or because it is able to create value in the business process that fulfils a customer requirement (Drucker 1955)? This is obviously not the case from an operations perspective, where the strategy needs to be based primarily around the business process while simultaneously considering the customer's needs. However, when the overall business strategy is based around financial data, with the overall aim of the activities based around profit maximisation, there is potential for difficulties.

Once the corporate strategy has been created that theoretically determines the best course of action for the company, it is then converted into a business strategy that is likely to be made up of a selection of business targets (see Figure 1.2). These may include the expansion of certain aspects of the business that have potential for greater profit and reductions in areas that are less profitable. The major problem with a process of this type is that apart from the lack of actual business information, there must be an extensive process of strategy dissemination, with the process being initiated from a single department. Not only does the planning department have to effectively communicate their work to the board, which may require considerable deliberations. The same process needs to be carried out when the corporate strategy is converted into a business strategy, where it needs to be approved and accepted by the business managers.

With the foundation of the strategy being financial, that is already looking backwards (Johnson and Kaplan 1987), combined with the time required to transfer such initiatives, once the information gets to the business processes, it will not be representative of the current business environment. This means that the targets that the business will be required to work towards may simply not be possible. Notwithstanding this, these are the targets that will determine if a particular function is successful in a particular year in the eyes of the board and the investors, which will in turn, determine the following year's plans. With such an approach to strategy, the long-term aspect of the work does not seem to be of particular importance, with the business functions doing whatever is necessary to meet financial targets. Figure 1.3 illustrates how this may affect an organisation's development over time, depending on functional dominances present within an organisation.

Although formulation of the overriding strategy is based around financial elements, the profitability of a particular business is likely to be defined by the operations that will realise the strategy. Taking a purely financial view of an organisation, there may even be a tendency for the operations to be considered a constraining factor or even a necessary evil. To convert the strategy into functional targets and aims, there are further 


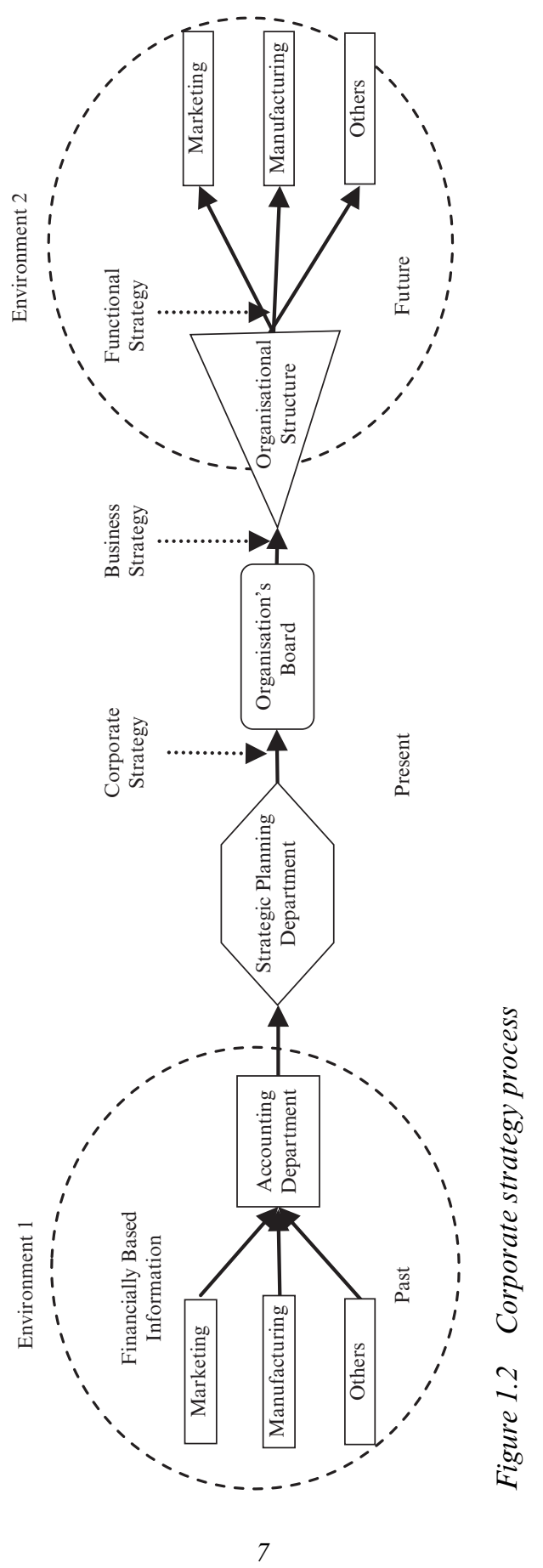

Kim Hua Tan and Rupert L. Matthews - 9781849802383 


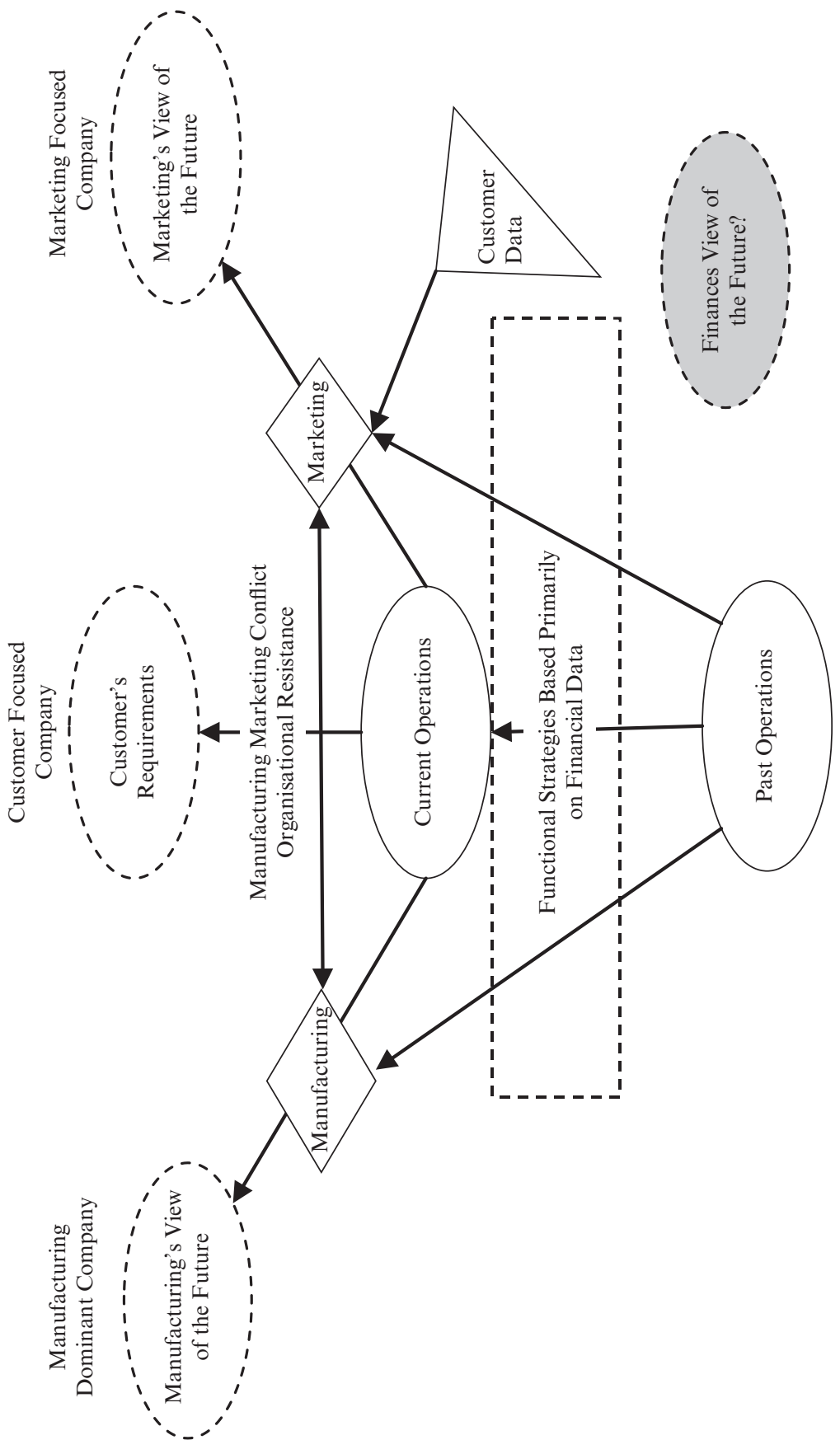

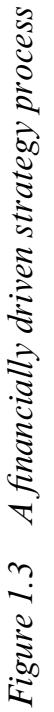


processes of dissemination, interpretation and conversion, to move from a corporate, to a business, and then to a functional strategy that aims to direct the functions in a way that achieves the higher-level business aims. However, due to the overall targets being based on financial data, the functional targets are unlikely to have guidance of how the targets are to be achieved. With a basis of profit maximisation derived from logic, rather than a more rounded understanding of the business functions, this may lead to business functions working towards business targets that do not consider the needs of the business. This is represented by the grey oval that illustrates how a financially driven organisation may develop itself out of business by not being able to meet the needs of the market.

\subsection{FUNCTIONAL APPROACHES}

In a traditional view of a company, all the different business functions have their own approaches and preconceptions of how to be successful in business. When business targets are based around the maximisation of profits, the functional approaches will all reflect this. Manufacturing will consider that the more time they are able to have their equipment producing, the more profit they will be able to make, meaning the primary aim must be to reduce unproductive time to a minimum and reduce associated costs to a minimum. A marketing view will be to sell as much as possible, by offering the customer what they want, when they want, so they are also able to maximise their volumes. However, if these are carried out in isolation, they will create considerable friction between the departments, due to aiming for different targets that are traditionally mutually exclusive.

The difficulty in both these situations is that the functions must achieve their targets to be considered successful, with no other constraints than a budget that is based on previous year's performances. Measuring performance in this simple way and driving developments in this way cannot be considered strategic, as there is no foundation for consistency. If companies give greater authority to marketing, this will lead manufacturing into inefficient ways of operating and companies directed by manufacturing will produce parts very efficiently but be unable to sell them. For this reason, over time business functions have begun to be considered more strategically, realising the introduction of consistency in functional developments is important for long-term company success. Considering the long-term impact of activities on the business, rather than basing all decisions on the financial information, has led the business functions to consider themselves in a more strategic manner.

For functions such as marketing, whose impact on the business is 
extremely difficult to quantify, with subtle aspects determining whether items sell, means this has greatly affected the approach of marketing. Rather than simply a selling function, they can act as customer representatives within the company to help develop long-term relationships. The traditional marketing activities such as advertising have also changed, by considering intangible elements that can build customer loyalty over time such as a brand that assists in reducing the need to determine a particular activity's contribution in a particular financial period. Other approaches include the focus on identifying specific markets that have potential for development into areas of future profit. Although here, the strategic aspect needs to consist of identifying future opportunities that are only possible by developing good working relations with the customers.

Although this development and the developments of the marketing function in general have improved strategic marketing, it is the development of manufacturing in a more strategic manner that is of more importance to operations strategy. Even so, as will also be outlined, both financially based high-level and customer-focused elements of the business are no less important within an effective operations strategy. By considering a company as a whole, rather than focusing on a number of different functions achieving their goals and creating a strategy that reflects this, there is potential for all areas to work together to achieve a single goal. Through understanding how all elements contribute to this goal, which is to create and satisfy a customer, this can be achieved without necessarily working against each other, where ideally the activity will result in profit. In this situation, even if there is not a profit, it may still be possible to consider the business a success by focusing on future developments that may not be at a stage where they are profitable but add intangible value to the company.

\subsubsection{Manufacturing Strategy}

Although the different business functions consist of very different jobs and activities, there is potential for each of them to have a dramatic effect on the business as a whole that can result in better financial performance. An area of early interest was manufacturing, once it began to be understood that measurements based on cost alone were not appropriate. Using a simplistic view of a manufacturing plant as simply making and selling functions, gaining a better understanding of how other functions could support the manufacturing element had potential to improve performance. This is possibly the reason why manufacturing strategy became a subject in its own right before other business functions but many years after corporate and business strategy (Hill 1985).

Historically, with such a fundamental view of a company, it was 
assumed that the more one was able to produce of any given product, the more profit it was possible to produce. If marketing was the function that determined if targets were met, the effect this had on the manufacturing department was to work to supply what marketing had sold in an appropriate way. With the amount of products that were sold seeming to have an obvious effect on profitability, marketing would be tempted to offer products in such a way as to increase sales. This may include such activities as volume discounts, low minimum order requirements or promising quick deliveries even though it may not have been possible for the manufacturing function to deliver. The result of this on manufacturing would have been many change-overs, short production runs and high inventory levels, all potentially strategic targets within the manufacturing portion of the business strategy.

With the marketing department potentially receiving more attention from upper management, the result of this would be manufacturing continual 'fire fighting' to meet orders that are received from marketing. This may lead them to spending a considerable portion of their time on business-as-usual activities, notwithstanding the fact that operating their function effectively required considerable time and effort. The addition of such disturbance from marketing, removes more of their time from being able to consider their business function in a strategic manner. However, an immediate effect of such activities on the business as a whole is great inefficiencies within the manufacturing function, which although translating to better figures for marketing, simultaneously reduce the profits for the company as a whole. In addition, requiring manufacturing to work against their strategic targets will introduce an element of conflict (Figure 1.3 , which can further reduce the ability to operate effectively.

From the traditional marketing view of production as simply the source for the items that are going to be sold (Wheelwright and Hayes 1985), the above situation is almost inevitable and made even more likely if members of the board have more traditional views that consist of marketing determining profitability. However, with alternate views of the manufacturing capability as proposed by Hill $(1985 ; 1993)$, manufacturing can gain the attention they deserve from upper management, as a way to improve the company's ability to compete. In the traditional model of manufacturing, it is thought that simply producing more will result in profit that in turn drives the marketing-driven view. However, as the understanding of the manufacturing function has developed, it can be appreciated that volume is not the only aspect that affects overall profit. By taking this perspective, it can be understood that focusing on different elements can improve profitability to a similar or even greater degree than volume.

Although it is true that producing more parts will reduce the unit 
contribution for amortisation of machines, machines generally have a fixed maximum capacity, meaning it is only possible to produce so many parts. Alternatively, by focusing on different elements that affect the cost to produce a particular item, it may be possible to achieve the same amounts of profit without having to manage the problems that can be associated with increasing volumes. This could be as simple as having to manage greater amounts of raw and finished materials or the knock-on effect of running machines for longer, such as the reduction in available time for maintenance. The problem is that the alternate approach to managing this element of the business would require considerable focus from the manufacturing function but also require appropriate support from the marketing function to be effective.

By focusing away from the volume approach of improving profit, possibly by identifying ways of reducing waste within the process through improving quality, changing design or even working with suppliers to reduce component part cost, profits can be greatly increased. What this requires from the business as a whole is an understanding that volume does not equal profit, and by possibly improving process technologies or other areas of manufacturing, profits can be increased. By focusing specifically on manufacturing strategy, Hill $(1985 ; 1995)$ aimed to educate the marketing board members about alternate approaches to success. Even basing these arguments on profits, which, as mentioned above, is not the best measure of performance, is potentially a starting point to improve 'congruence of purpose and function' (Hill 1995, p. 55) by describing the effects in a suitable language.

As much as anything, the goal of Hill's work as a whole seems to be unifying the company, with concepts that transcend the organisational boundaries. With better understandings of the manufacturing function and by selling smarter rather than harder, it is possible to reduce the trade-offs where both departments seem to be working against each other, while simultaneously providing the company with better profits. However, unless the company's strategic plans are altered in an appropriate way to reflect this change in focus, there will still be a tendency to revert to the old way if they are continually directed towards meeting their traditional functional targets. The above approach to manufacturing strategy, although different from more traditional approaches to strategy, is still very much focused on profit maximisation, rather than being particularly focused on achieving a particular global strategy. However, this is just a potential approach to a manufacturing strategy and could be achieved with activities that are strategic, representing long-term consistency of aims.

An element of the above approach that does have potential for longterm improvement is the focus on marketing/manufacturing links, that 
could form the basis of improvements not based entirely on financial results. By improving relations between marketing and manufacturing there is potential for requirements of the customer to be considered when improvements are being made within manufacturing. Rather than marketing-driven manufacturing, there is a shift to 'customer-driven manufacturing' (Berry, Hill and Klompmaker 1995): by considering the customer during product and process developments they can be done to reflect the needs of the customer rather than the needs of a business function. In this situation, as with other elements of business, it is also important to consider the long-term implications of investment; even though investing in 'cash cow' products may make financial sense, decisions should not be made without considering other measures of performance.

Although the above approaches to manufacturing strategy consider developments in a slightly more strategic manner, they do not necessarily take account of the longer-range elements of strategy. This overall strategic vision is still required from the board so there can be consistency for the whole company or group of companies. However, the development of these approaches to other business areas allows for potentially better results that will keep the customer satisfied. Unlike the focus on marketing, within manufacturing, the need for new concepts is possibly less, as simply directing process improvements has the potential to return considerably more tangible benefits. Manufacturing is not without its own developments although it is considerably more practical and more likely to consist of getting the business-as-usual activities perfected. Even so, by combining a number of areas of development, more developed manufacturing strategy concepts can be created, that better reflect the needs of other business functions that promote lasting satisfaction for the customer (see Figure 1.4).

\subsubsection{Transitions to a More Developed View of Operating}

During the same period that the above approaches to developing manufacturing capabilities were presented, the competitive environment began to change with increasing competition in the western markets from eastern manufacturers. During the 1980s, with the appreciation of Japanese approaches to manufacturing, along with western alternatives, initiatives were introduced into manufacturing companies with the aim of improving quality to be able to remain competitive in global markets. The shift in focus required by upper management to understand the significance of focusing on manufacturing capabilities was actually only one aspect that needed to be considered when taking such a different approach to business. With the eastern businesses having a deep appreciation of the 


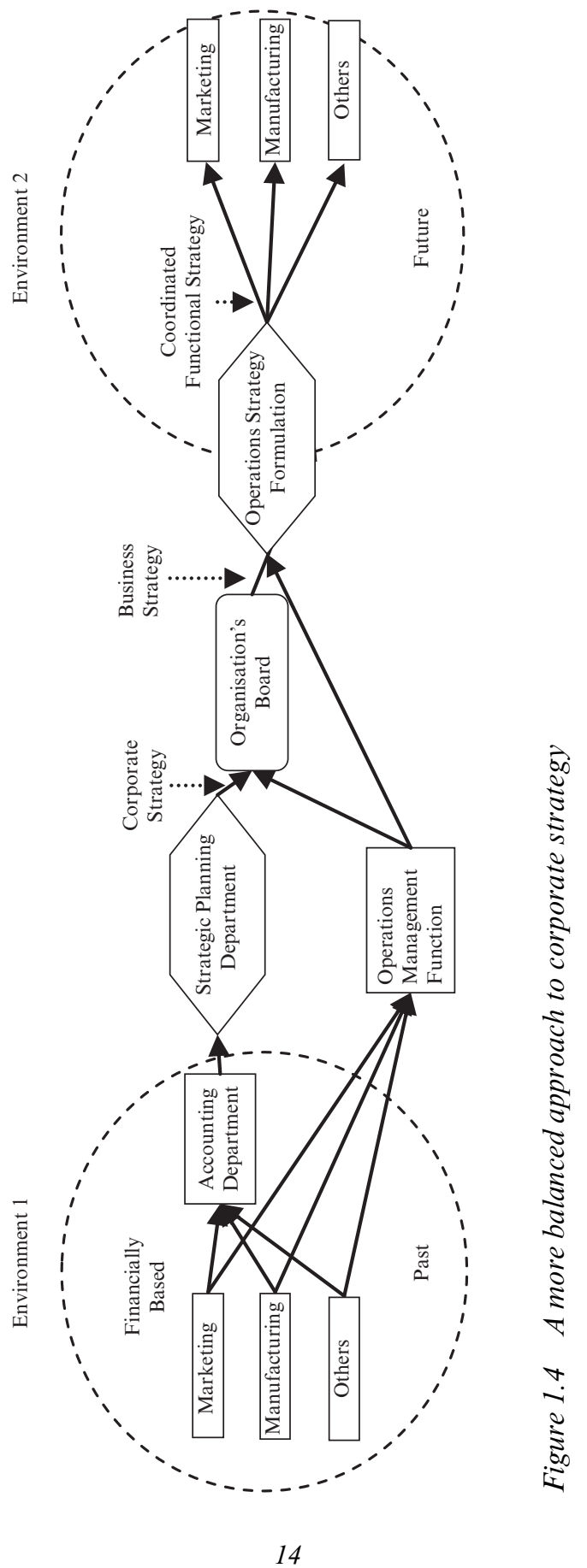

Kim Hua Tan and Rupert L. Matthews - 9781849802383 
systems they had developed, they were able to appreciate manufacturing's ability to meet customer needs by innovating as well as producing.

During the annual planning sessions of upper management, the reductions in market share and rise of companies offering improvements in all areas of business performance made such a change in approach seem necessary. The inherent problem in such a situation was its top-down, hierarchical nature, where ideas needed to be passed down and enforced. Altering approaches to manufacturing and marketing, although requiring a certain amount of change in culture to take place, did still fit in with the western way of business. Although management would have to change the way they carried out their strategic activities, it was at least possible to justify such activities from a financial perspective. The more difficult part of such a change in approach to strategy was not simply changing the aims and targets given to the company, but changing the approaches of the company to allow for generally better coordination. With the developing, but still traditional views of strategy, the introduction of such initiatives was still carried out in a functional way which would not help build the level of coordination that was well established in the east.

With the obvious financial benefits of the different approaches to manufacturing of lean (Bicheno 2004) or the Toyota Production System (TPS), choosing to adopt such an approach to running a business would, and for many companies did, seem like the right choice. Unfortunately, with the traditional, top-down, functional approaches, the total change required in the business was much greater than management understood, meaning that the support that may have been given with budgets was not matched with bottom-up training. Where the western approaches to more developed functional strategies required an amount of coordination, the eastern approaches required a business to be managed as a single business function. In these situations, if the upper management were introducing these initiatives with financially driven motives and only low levels of understanding about the approaches themselves, problems were almost inevitable.

Considering such initiatives as ways to increase profit and general performance instead of as a result of a deep understanding of the philosophies that allowed them to produce such benefits is a possible reason for so many failures in western business. The reason for their success in the east was due to a different approach of all staff and by considering management in a different way; this reduced the top-down nature of such initiatives, where management assist and facilitate rather than control and enforce. The new and fashionable approaches to business improvements required a 'bottom-up' approach to business that was grounded in business-wide understanding of what the total aims of the business were. 
With a 'top-down' introduction, focusing on business results instead of customer-based strategic aims that should result from the initiative, other areas of the business were not developed in a suitable manner in order to promote and nurture a suitable culture.

The initiative, when viewed from a financial perspective without a deep understanding of concepts combined with a traditional functional view of business, would have considered the initiatives to be primarily manufacturing related. If this was reflected in how the approaches were implemented, the lack of support from other functions created may also have affected the approaches' effectiveness. The result of this may have been resistance to the initiatives that seemed to offer such obvious benefits when viewed in companies where these initiatives had been invented and the company-wide culture had been established. The problems were then magnified when considered from a financial point of view, such as return on investment, that would have been made worse by the resistance from other functions that may have seemed to have fallen out of favour with upper management. With a better understanding of the initiatives, it would have been apparent that the benefits from these new approaches to business resulted from the combination of all the areas of business, which may have been considerably more difficult to quantify in short-term financial measures. Many companies introducing such approaches would have soon realised that simply reducing the level of inventory in a warehouse did not reduce costs or improve performance, without other systems present that supported the needs of a low inventory system.

Unfortunately for many 'champions' of such initiatives, who may not have had such an understanding, when results did not noticeably improve following the introduction of new working practices, investment would have been reduced or even removed. What was probably not understood to a suitable degree was not the mechanics of such initiatives but their strategic nature with regards to the whole company. There needed to be a business/corporate strategy reason to introduce an approach that would have such a large effect on the way the business was run, rather than simply a cost reduction exercise. By understanding this, the strategic goals could be aligned with the introduction of the system, so that during the implementation, cost information was not the only gauge of performance. This would mean the performance of the initiative would not have just a single planning cycle to show a financial return, that if negative had potential to reduce support. Such targets could be to align business functions with a consistent approach to improvement, or could reflect the service that the customer was receiving.

Appreciating the reason for introducing such an initiative is not wholly internal and functional but that it should represent the needs of the whole 
company is very important. In a traditional western company, this could consist of all parts of the business strategy being consciously focused on achieving a specific business initiative (see Figure 1.5). If this is carried out successfully, all elements of the business will work in a way that is consistent with other areas of the business and help to achieve the business goals. The results of such a directed and synchronised strategy process could be consistent actions in all areas of the business, which has the potential to reduce the resistance mentioned earlier. The alignment and consistency between all departments (or business units) working towards a single goal could also mean that the financial effect of many coordinated improvements would be more noticeable, assisting in maintaining management's enthusiasm for the initiative. If the improvements also resulted in improvements that were directly noticeable by the customer, for example if marketing received positive feedback from an engineering activity, they may be more considerate to the needs of that area of the business.

Although initiating a western approach to process excellence, General Electric's (GE's) adoption of Six Sigma is one of the better examples of how such a change can be achieved while also demonstrating how very difficult it can be. Six Sigma is a statistically based approach to reducing process variation devised in the west to compete with eastern approaches such as Total Quality Management (TQM) whose heavy reliance on bottom-up activities created problems in some western implementations. The approach focused more on management's responsibility, an important element of Joseph Juran's teachings, but combined it with the ideas of W. Edward Deming, another very influential quality thinker; that reducing variation would result in reduced cost. The result was a fact-based, statistical approach that appealed to managers due to its structured nature but also to finance with its aim of reducing costs that resulted from the reduction of variation. Another reason for its appeal is that, unlike other approaches, it is specifically applicable to non production, administrative activities, which helped to expand the scope of the strategic initiative.

Even though Six Sigma westernised aspects of eastern approaches, it does not remove all of the difficulties faced during its introduction into a company, but assists in improving support. The problems that remain are achieving suitable understanding in enough members (a critical mass), while also converting this understanding into company-wide results. In GE this was possible due to the leadership of Jack Welch, who understood the potential benefits of the approach and had the ability to gain backing from all areas of business. However, on his own, leading from the front was unlikely to have been enough to guarantee success, which meant there was a need to introduce company measures that supported implementation, such as the alignment of the human resource function with the 


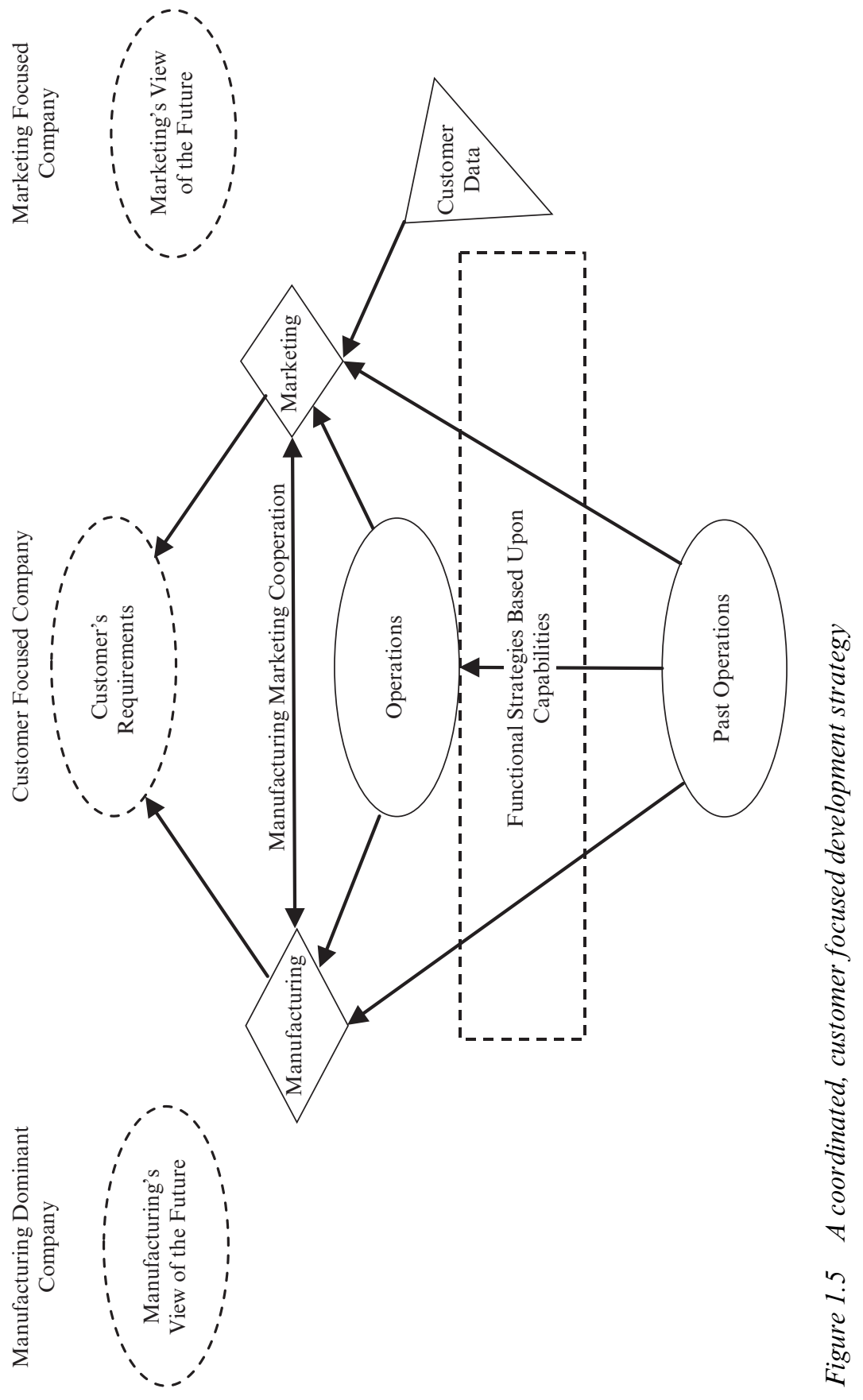


initiative. Even though the approach could be fuelled by heavy investment in Six Sigma training of 'belts' (specially trained statistics experts), the support from other business functions to support the process champions also aided the initiative's success.

Understanding that successful adoption of the initiative offered an opportunity for GE to develop a sustainable competitive advantage, rather than simply reducing cost, was a reason Jack Welch managed to change company culture. Six Sigma, as a corporation-wide initiative, also represented a common strategic aim for such a diversified organisation, moving focus away from direct financial management, understanding financial success is the result not the driver of achieving the strategy (Drucker 1955). Altering the view of an improvement initiative from one focused within a particular area of the business to one that is relevant to the whole business is an important transition for both the subject and its use in practice. It reflects the need for cross-functional improvements that were mentioned in the functional, manufacturing approach to converting corporate strategy to business improvements.

\subsubsection{Still Room for Improvement?}

By reviewing approaches to improvement activities in relation to the overall business strategy, we have attempted to demonstrate the importance of considering these improvement activities strategically rather than simply financially or functionally. Even though the significance of such changes seems obvious enough to prevent conflict and build support, a change in how complex initiatives are approached has not been widespread. Demonstrations of how it is possible and that it is not necessarily the approach's fault for failure, such as GE, have also not assisted in changing the way organisational development is managed to improve effectiveness and general execution. Research outlines the need for a change in approach to such initiatives that helps to change and support the development of both cultural as well as more tangible changes.

This is reflected in the continued development of new production techniques that aim to remove the problems experienced with other, previously fashionable approaches to improvement. A highly regarded approach that is able to give results many times better than Six Sigma, lean and TQM is the Theory of Constraints (TOC) (Goldratt and Cox 1992). In a similar way to GE's implementation of Six Sigma, TOC works on the understanding that it is the whole system that determines success so it is not appropriate to introduce improvements to a single area of the business. Differently from Six Sigma, TOC understands that improvement made in areas that already have operational spare capacity (slack) cannot give improvements 
in performance to the system, so that the most efficient way to improve performance is to direct improvement to the system's bottleneck process.

The result of this is that by focusing improvements in a much more directed manner, the returns on investment should be improved significantly and, where successful, results are considerably more effective than similar implementations of other improvement initiatives. However, considering such an initiative from a more strategic perspective does bring up questions about whether it is as applicable as other approaches as a means of developing an effective company strategy. The reason for this is the basis on which TOC is established, which is that the purpose of a business is to make money now and in the future (Goldratt and Cox 1992). In regards to strategic activities, such a foundation has the potential to give additional weight to the financial approach to company management, even if it does promote the achievement of global rather than local goals.

Unfortunately, with the financial perspectives, such an approach also needs extremely careful strategic management to allow it to assist company operations as a whole, rather than just making it more profitable in the short term. Due to focusing on improving the company's ability to make profit, there seems a greater possibility to lose focus on a different aim of a company, that is to create and satisfy a customer. The effect of this could be the selling of one's future to succeed in the present, by maximising profit within the current market without looking forward and developing capability for the future. However, if in introducing such an approach to business a practitioner considers product and process development in the same context as the production system, the risks associated with such a system may be reduced.

With the development of an approach such as TOC to improve business performance, although the problem of improving business performance may have been solved, it is still the introduction and effective management that really determine if such an initiative is a success. This may be a reason for the subsequent developments of the TOC approach that enabled the ideas to be transferred from operational level-development to assist in specific strategic activities. As well as the continued fine-tuning of the TOC approach to take account of the need for strategic elements, there has been the development of a subject that aims to take account of these issues: operations strategy. Without focusing on a particular area, other than the operations element, operations strategy aims to introduce more practical aspects to the subject of strategy to assist in converting high-level strategic ideas into improved business activities without focusing on a specific business function.

The next section gives a brief outline and introduction to the subject of operations strategy to show how the above approaches to strategy have 
contributed to the current thinking of this approach to strategic activities. Within this subject the focus is away from a corporate, global vision of strategy and also away from the marketing elements; however, these still need considerable attention. Although the above approaches to these elements of strategy have a heavy financial bias it has been the aim to outline the need to move away from such a starting point. The reason for moving away from such an approach, apart from its potential irrelevance and self-perpetuating nature, is that strategic planning from a financial basis does not necessarily have a long-term element. Without an element that represents consistency from one year to the next, a strategic planning process based on such information cannot be considered strategic, as business targets can change from one planning period to the next. Although operations strategy does appreciate the need to consider financial aspects, the above outlines the need to consider it as one of a number of measures that drive developments and customer satisfaction.

\subsection{THE NEED TO DEVELOP AN OPERATIONS STRATEGY}

As outlined above, a single area of a business cannot be the focus of strategy and it is only when the scope of a particular type of strategy is expanded to include more elements of the business that it is able to reflect the needs of the business. Such thinking is in line with the view that it is also not a particular function that allows a business to be successful, but the way they all work together. Moving away from a functionally based approach to the management of a firm gives weight to the argument for the development of operations management capabilities that are able to coordinate functional capabilities more effectively. However, the focus of this type of work is at an operational level of business, concerned with the day-to-day running of a business rather than longer-range developments. For this reason, to reflect a broad view of a business, there should be a broad view of strategy that considers and integrates the different elements, rather than simply allowing for the needs of different areas of a business.

The foundation of such an idea of business and strategy is not new along with proposing new ideas of business as a whole, Drucker (1955) proposed a different, more rounded approach to directing a business's development. As stated earlier, financial measures represent the result of efforts but should not be the only measure and cannot be used to drive improvements. For this reason, Drucker proposed a management-byobjectives approach to business that tracks business performance and 
development with seven distinct measures that although including financial measures understand the need for a more cohesive approach. Even though the ideas are very relevant today, such ideas were potentially ahead of their time but also, the business environment at that time did not require such approaches. In the 1950s, applying the technology that was available in a way that allowed profit maximisation was the major focus, and due to the stability of the environment in general, the risks associated with pure financial planning were fewer.

It was not until 1987 that such questions were raised again, to be heard by business professionals that management accounting alone was no longer an appropriate way of guiding a business (Johnson and Kaplan 1987). In a business environment that had many more examples of businesses that had been managed with the exclusion of other relevant business data to their detriment and demise, the need for a different way to manage a company was greater. This is potentially what formed the basis for a more operational view of business strategy and the development of operations strategy as a specific area of academic and professional interest. However, unlike Drucker's approach, the altered view of how to control a business was followed up by a relatively simple tool to integrate and present these old ideas in a new, manageable form. The Balanced Score Card (Kaplan and Norton 1992) measured the performance of a business in four areas that did not just show where the company had come from (financial) but also its current position and where it was going (see Figure 1.6).

With business operations being seen as an area with potential to offer a business a distinct competitive advantage through its effective management, a strategy that reflects such a coordinated business function also needs to include all relevant areas. In the above approaches to strategic management, the need to consider the business as a whole is outlined, to assist in reducing risk while also giving specific attention to long-term improvements. For this reason, operations strategy as defined by Slack and Lewis (2001) needs to represent four major areas of operations to aid consistent management of such an important aspect of the business entity. In the more traditional views of business, such an approach to strategy may seem like the manufacturing strategy approaches, although the operations that take place are not necessarily production. The operations could be any type of business, not necessarily manufacturing, but rather the element of the business where the transformation process takes place.

By considering operations as the transformation process rather than simply the manufacturing plant, which is the focus of manufacturing strategy, the scope of the strategy needs expanding in an appropriate way. As well as including the strategies involved within a given function, it 


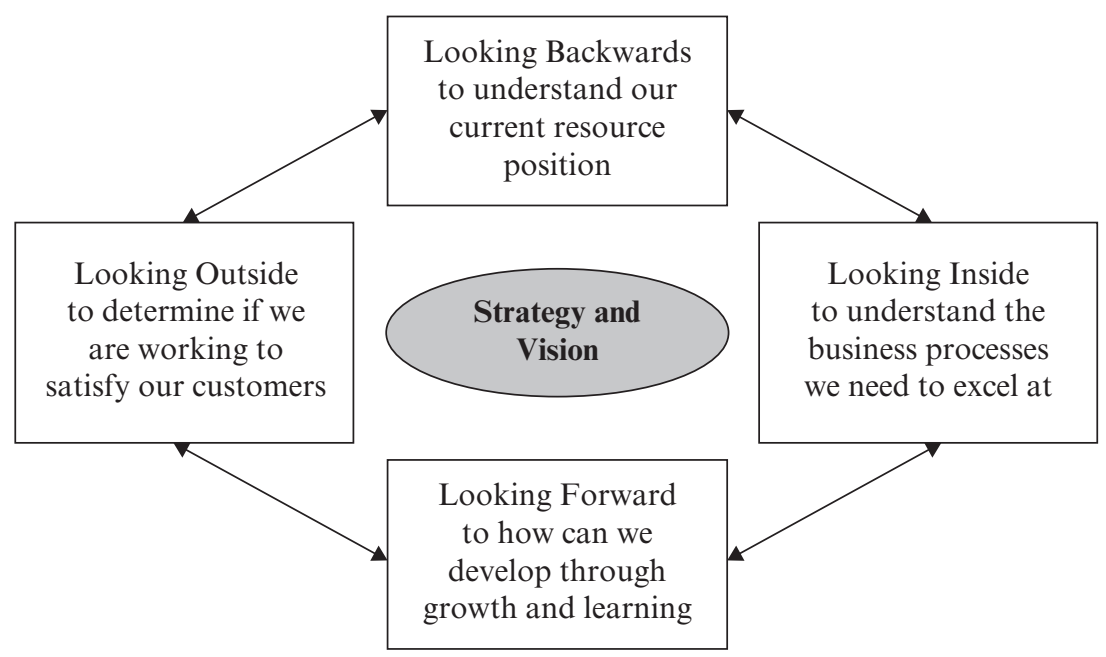

Source: Adapted from Kaplan and Norton (1996).

Figure 1.6 A balanced score card approach to performance measurement

must include higher-level transformation processes such as inter-business interactions within a supply network while also including low-level process improvement strategies. In addition to considering different levels of the transformation process, operations strategy also needs to be able to consider different types of transformation processes such as service and even charitable organisations, as the aims of them are all essentially the same. Within this setting, the need for operations managers is still present in order to improve performance, but operations strategy is required to direct their improvement activities in an appropriate way.

Slack and Lewis (2001) define operations strategy as 'the reconciliation between market requirements with process capabilities', reflecting both the need for marketing strategy and manufacturing/process-based strategy. This definition demonstrates the reason for already having touched upon both marketing and manufacturing strategies, showing the way both developed to reflect the needs of the business. Operations strategy also needs to reflect the overall corporate direction of the company, to prevent other business functions leading it away from the needs of the investors. In addition to these three areas, it is considered necessary to listen to the voice of the company, to enable the engagement of the company as a whole and learn from the experience they gain from carrying out the transformation process (see Figure 1.7). 


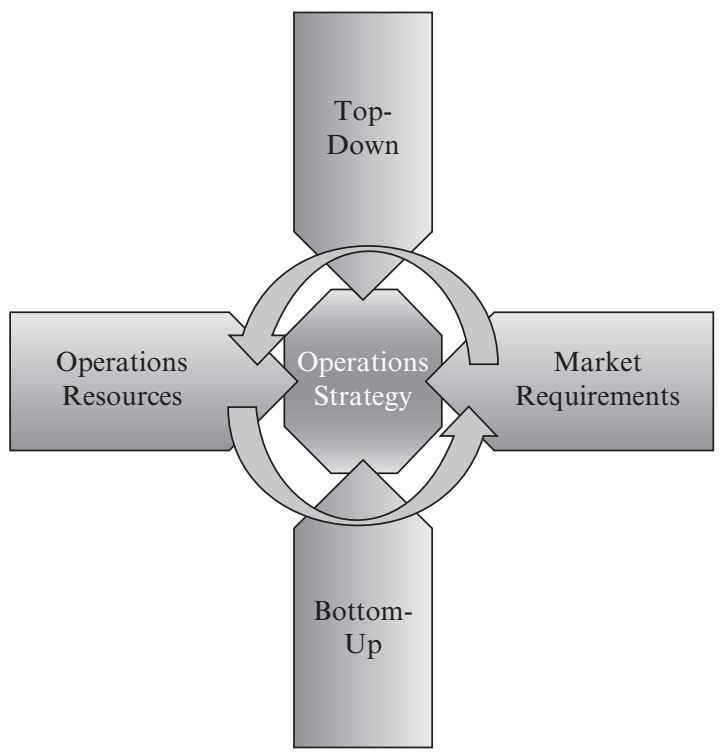

Source: Adapted from Slack and Lewis (2008).

Figure 1.7 Contents relations to operations strategy

\subsubsection{Top-Down}

The top-down approach to strategy reflects the traditional elements of corporate and business strategy, where the board or a strategic planning department determines the overall direction for the company such as divesting and acquiring certain business units. Unlike the traditional topdown approach, such information contributes to the operations strategy, rather than being the basis for other types of strategy. This ensures that the strategy that drives the development of operations is consistent with the requirements of the board and investors. In a diversified corporation this is particularly important, as it ensures business cohesion to maintain corporate identity. This may also be where a corporate initiative is formulated that forms the basis for further strategic activities across the business.

\subsubsection{Bottom-Up}

Not mentioned directly earlier, bottom-up approaches rely on the experience that is acquired from the day-to-day running of the business to contribute to strategic activities. Originally identified by Mintzberg in 
1978 as 'emergent strategies', such a strategy is not implicitly formulated and then implemented; by learning and developing continually, strategy can be formed and realised (Mintzberg 1978). Learning in this way allows the business both to react quickly to localised situations without the constraints of a hierarchical system while continually developing capabilities to reflect the needs of the customer (Johnson and Medcof 2007). Unlike the other approaches to strategy already discussed, this specifically allows greater involvement of the company as a whole, as it is not reliant on top management or functional professionals to carry out strategic activities.

Such elements are particularly important within certain process improvement activities, such as TQM or TPS, as the responsibility for improvements is placed on this area of the business. Although Six Sigma involves similar type of activities, the presence of particular professionals or 'belts' to take responsibility means the emergent elements of this particular initiative are fewer, although a suitable culture is still important. Such an approach has been found to be, and still is, very successful in certain business environments, but it is considered difficult to initiate such bottomup activities within a company that is able to maintain a 'consistency of actions'. 'Consistency of actions' is particularly important when pursuing a wholly 'bottom-up' approach, as without this there may result uncoordinated improvement activities. Development of a business culture that is focused upon improvements that relate to the customer's satisfaction is a possible solution to this, where all actions can be considered individually to determine whether they assist in reaching the organisational targets.

Including a bottom-up approach to strategy requires operations strategy to specifically consider the day-to-day learning process in the development of a strategy. This not only assists in improving involvement of the company as a whole, but it also enables strategic activities from more levels of the company that allow for a smoother and quicker transition between the formulation and implementation stages of a strategy. Bottom-up information also assists in giving those charged with working on operations strategy better process-based information. Not only does this have the potential to allow a more appropriate strategy to be formulated, but it also means that functional staff are involved in strategic activities, improving their commitment to the process. The reason for this is, as they have been consulted regarding their specific area of the process, the strategy should reflect their specific needs, in essence being partially their own strategy.

\subsubsection{Market Requirements}

From Drucker's (1955) definition of the aim of a business, the view of the client/customer must be carefully considered in any approach that aims to 
improve the performance of operations. As with more developed views of the marketing function (Thorpe and Morgan 2006; Ranchhod and Gurau 2007), the element within operations strategy that considers the market does not consider what can be sold, but instead what is required. As well as considering the types of products that are required by the market, it is the source of the information relating to what is required of these products. The marketing function could be considered a representative of the customer that determines how well the company is performing, by being the source of customer satisfaction, possibly in terms of standard performance metrics such as Quality, Speed, Dependability, Flexibility and Cost.

It is also the function's responsibility to determine how well the developments allow the organisation to perform in relation to the market as a whole, such as more traditional marketing measures that determine market positioning. By understanding the current position in relation to competitors, possibly in specific areas of performance, there is potential for improvement activities to be focused to make the biggest effect on overall customer satisfaction. The market is also the source of information relating to the actions of competitors that can potentially affect the competitive position of the company if the company is unable to react effectively. Fundamentally, the market function is present within the operations strategy definition to ensure that improvement and development activities that consume considerable resources are directed to result in better meeting market requirements.

By including the market requirements in the operations strategy, the other areas of the business are able to appreciate and obtain relatively direct information relating to who is widely regarded as the most important element in the organisation. The effect of this, as mentioned above with manufacturing strategy, is that process improvements can be directed to reflect the needs of the customer. The upper management are able to appreciate customerspecific requirements that will assist them in being able to formulate an appropriate high-level strategy. For the bottom-up perspective, understanding the market requirements at a relatively low level potentially represents the source of consistency that is required to make an emergent strategy possible. What is important when considering all these areas together is that all externally facing measures of performance will be relevant (Johnsen 2001). This helps to ensure that the result of the operations strategy process will be an operation able to satisfy the customer more effectively.

\subsubsection{Operations Resources}

Unlike the manufacturing strategy view of operational development, by taking the view of the transformation process, operations strategy's scope 
is considerably wider. Considering the transformation process as the unit of analysis means the content of operations strategy is much larger, both including the manufacturing elements as well as higher-level inter-business relations. By taking an operational resource-based view of a firm (Jenkins, Ambrosini and Collier 2007), it can be appreciated how it is the company's inherent ability to combine resources into capabilities that is the source of its intangible market value. It is an operation's ability, not only to add value, but also to develop the value it adds that is specific to the company that allows it to continue operating in a competitive market both now and in the future.

With a considerably wider view of the operations functions than manufacturing strategy, it is not possible simply to focus on a particular area of the business to improve operations. This means that the content of operations strategy is considerably larger than the functional approaches to strategy and it is specifically the operations resources portion that is expanded the most. When considering such initiatives as lean or TPS from an operations strategy perspective, it can be seen that these are not in fact simply manufacturing but operational initiatives, and, as mentioned before, unless the scope of the initiatives is expanded suitably to include all operations, they are less likely to be effective. Within TPS for example, operations strategy content, such as capacity management or supply network, plays significant roles in the initiative. If implementers simply focus on developments with the manufacturing elements by reducing inventory levels, it is not possible to realise the benefits of such an initiative that are enjoyed by those who developed the systems.

Where the operations strategy view of operations resources is fundamentally different from the previous descriptions of process and operational development activities is that it is essentially focused on the development of a company-specific strategy. By understanding that what defines a company's capabilities in business are the resources it has at its disposal, and through the consistent development of these specific resources, it will be able to meet its own objectives rather than those of a historically successful company. What also needs considering is the directed development of the company-specific intangible resources that when developed enable specific jobs or services to be provided that cannot simply be purchased (Barney 1991). The ability to offer a customer a company-specific product or service is effectively the manifestation of the capability to combine tangible and intangible resources in an effective way. Through the structured development and acquisition of appropriate tangible resources with the appreciation of their ability to provide the customer with what they require, the activities can be structured appropriately to develop capabilities that are required by the business. 
The final element of consideration regarding the operations resources area of operations strategy is the operation processes. Although the resources represent the components of the business and the capabilities represent the systems, without an understanding of the processes that take place within the business, it is not possible to properly appreciate their relevance to the whole business. Understanding the processes present in a business also allows developments and improvements to be made with them. By carrying out such activities as business process visualisation, the relative importance or associated risk of certain processes can be identified for re-engineering to focus improvements in the elements present within a process (Pryor et al. 2007). For companies where specific activities are particularly important, the identification and optimisation of these processes are essentially similar to taking a Theory of Constraints approach to process improvement.

With the operations resources element of the business being where the majority of the transformation processes take place, its inclusion within operations strategy is obvious. Developing a strategy that gives specific attention to the area of business that carries out the important transformation process places focus upon the element of the business that will be responsible for the strategic changes. This approach also considers how the other elements of business can alter their approaches to support operational changes by learning from and directing further operational resource changes. Including the operations resource element in operations strategy allows the organisation to consider primarily what the company is capable of, such as what it can produce now and what it is potentially able to produce in the future. This means that when developing a company-wide vision, upper management will be able to do this in line with the specific capabilities of the operating function. Greater understanding of the operations function could even drive specific corporate strategy to direct particular investments into the further development of critical operations resources.

The operations resources elements are also very important for the other areas of operations strategy. It relies on the bottom-up approach to strategy so that the capabilities of the operation are able to adapt to in-process learning so that it can continually develop the process from the experience that is being accrued. The operations resource, as well as relying on the resources present to be able to realise developments, also requires input to take account of external factors that may need considering. The major benefit of including these two elements in operations strategy is that, while representing internal developments, they also carefully consider the views of those ultimately affected by developments. Without including these elements in strategy, it may become more difficult to realise innovations if they do not have greater appreciation of operational issues. 
As mentioned earlier, understanding the links between the manufacturing function and the marketing function, when considering the overall strategy, has great potential to reduce conflict while simultaneously improving company performance. However, the operations strategy approach, as well as taking a broader view of the operations resource function, also has a broader view of the relationship between these two areas of business. In addition to marketing selling in line with the capabilities of the operations resources, it is also possible for there to be considerable two-way learning. The operations are able to develop in a manner that is consistent with the market requirements, while also being a source of information about further requirements of the customer and how operations should begin to prepare for these needs. Such interrelations can form the basis for particular elements of the operations strategy that direct the development of particular areas of the business to enable them to remain competitive in markets that are not yet present.

\subsection{CONTENT OF OPERATIONS STRATEGY}

Although an operations strategy that gives consideration to the above four areas is likely to be of more use than one that does not, it is however likely that the majority of attention will be paid to the operations resource element. Although the contribution of the other three areas is very important, it is likely that their input into the actual activities will be more of consideration rather than direct attention, with the actual work remaining within the different functions. This effectively means that paying specific attention to corporate strategy is still as important as with other approaches to strategy, although with an operations strategy approach corporate feeds into it, rather than being the sole driver. The same is also true of marketing strategy; although consideration is given to the marketing function, operations strategy is not concerned with traditional marketing functions such as advertising campaigns. Even so, the presence of a traditional marketing function with its own marketing strategy is just as important, if not more necessary today than historically, with the increase in the competitive environment. However, the marketing strategy does need to reflect the needs of the operations strategy, by developing approaches that assist in directing appropriate information into the operations function. The changes in corporate and marketing strategy when considered in relation to an operations strategy are to consider them both in a wider context, to effectively stop short-term measures, in effect, making them more strategic.

In this respect, the content element of these two aspects of operations 
strategy remains relatively unchanged from a more traditional view, simply including additional information. The fourth area of operations strategy, bottom-up, is, by its very nature, very different from the other two, being an observed phenomenon rather than an area of specific strategic attention. Even though focusing on developing this area can allow for important emergent elements of strategy, the way these strategies come about means it is not possible to study and direct them as that would mean they were deliberate rather than emergent. Without being able to study and direct such an approach to strategy, a different element of business becomes of interest, whose study and development help promote such emergent strategies. The concept of a learning organisation is very important in today's highly competitive market; without it, the learning may be restricted to particular professionals or functions. This effectively maintains elements of hierarchy within the organisation, which can resist change, reducing the organisation's ability to quickly identify and meet market requirements. Although this area of study affects the other three by effectively enabling the company as a whole, it is also not specifically studied within operations strategy, although its careful consideration is beneficial.

The remaining areas of operations strategy, operations resources, is the area where the majority of attention is directed when developing an operations strategy. This reflects the progression of manufacturing strategy into operations strategy, although as already mentioned the range of elements included within an operations strategy is considerably larger than the manufacturing function on its own. As with the three other areas of strategy, the most important change is not necessarily the increase in the amount of business elements it considers, but rather the way it considers them in relation to the other areas of business. However, even though it may be the interactions between the different elements that will determine excellent performance and offer sustainable competitive advantage, there still needs to be focus on the specific areas of operations, meaning within the study of operations strategy there must be substance and content.

As outlined above, focusing on almost any area of transformation and developing in a way that reflects the needs of the market are potential content. These areas include focusing on improvements at different levels of the business from introducing changes in a frontline activity to analysing the route products take along a value chain to identify and better understand the areas that require improvements. The following subsections will give a brief outline of some of the different elements that make up the content of an operations strategy, working from low-level processes such as directed performance improvements to high-level supply chain network strategy. Once these areas of operations strategy content 
have been discussed, there will be a section on operations strategy process, which is the activity of using the content to create meaningful strategy to be implemented within an organisation.

\subsubsection{Process Improvement}

Using the term 'low'-level improvements gives this area of operations strategy content relatively low importance, when it has anything but. The 'low' simply refers to the level in the business where these activities can (although not necessarily do) take place. The performance of an organisation is generally determined by its ability to carry out activities that are deemed important to the customer. These areas may be generic performance measures such as cost, quality and speed, although depending on the business in question, the generic measures of performance may be replaced by more company-specific measurements. This is to prevent ambiguity while helping to direct improvements, for example a measure such as speed will have very different meanings for a logistics company and a product development office. For this reason, more company-specific performance criteria may be more appropriate, such as order response, delivery lead time or time to market.

Using marketing information that is available to the process assists in directing improvements in a way that will affect customer satisfaction; combining this with a performance management system may further help to direct process improvements (see Figure 1.8). Such an approach to process improvement is particularly important when considered against traditional constraints in manufacturing such as trade-offs. Careful understanding of the acceptable levels of certain aspects of performance can

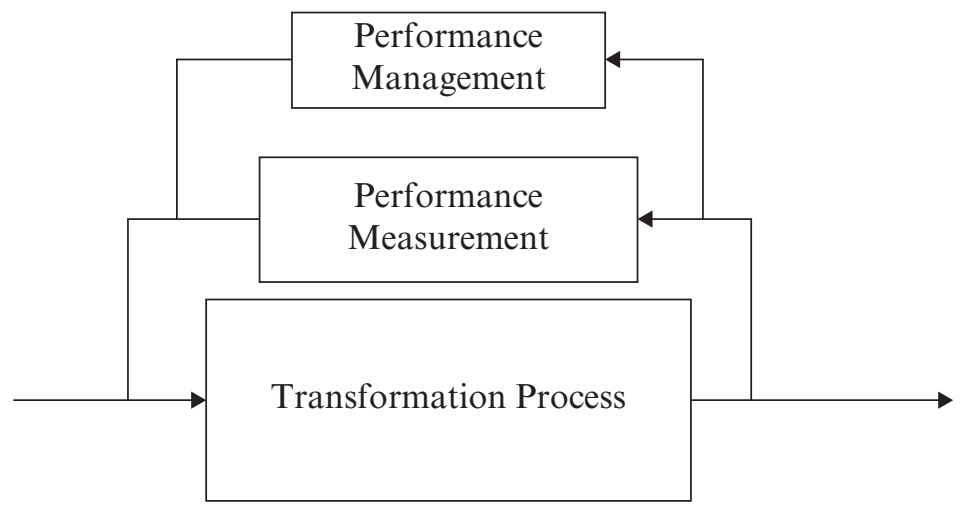

Figure 1.8 Performance measurement/management 
allow improvements to be made that give the best overall improvement for the customer. Even though it has been possible to reduce the direct effects of manufacturing trade-offs with the use of more developed approaches to manufacturing, prioritisation of improvements is still important. Understanding how the resources available for improvements can be assigned to give the greatest improvements in customer satisfaction represents a way of maximising return on investments.

However, due to the constantly changing market place with new products, suppliers and even solutions to problems, the need to continually monitor performance criteria is established. Over time, even the performance criteria of a single product will change, and depending on the industry in question, may change very rapidly, such as consumer electronics. For this reason it is not simply adequate to focus on the process and products that are present in the market; it is essential to understand how different levels of process performance are able to change one's position in a market. As well as performance criteria changing over time, the product elements required by the market change over time, meaning a constant need to develop a product, but also a need to develop new products that reflect the need of the current and future market. The developments made in frontline activities are an important source of information that can affect a company's performance directly; they are also an important source of information that can be considered in the development of process technologies.

\subsubsection{Process Technology}

As mentioned earlier, although improvements can be made to an operating system to meet the needs of a customer, the processes used to make a product are likely to limit the extent to which these changes can affect performance. Not only is it that a particular process is unable to meet particular criteria in volume, quality or flexibility, it may simply not be able to produce a product. This means that an element of a product that is just as important as the product itself is the processes that produce it; without a match between the two, important market criteria will not be met. For example, a change in process technology, such as that witnessed in the automotive industry, can change the product entirely from a luxury item for transporting the elite to part of a basic existence. Although this example is one of the more extreme, the same can be true for much smaller changes, such as the development in robot laser welding improving car safety standards.

In certain industries, such as the oil industry in particular, the product and process are effectively interlinked, meaning that in certain instances 
it may be necessary to actually develop a market for a product. In other cases, such as the electronics industry, the importance of developing a manufacturing process as well as a product is extremely high. Without the ability to produce a product in such a way that there is a market for it, there will be no process for recouping the costs associated with the design and development activities. However, with the possibility of licensing developments, for others to develop a process there are alternate way to recoup research and development (R\&D) costs, but unless the focus of the business is $\mathrm{R} \& \mathrm{D}$ rather than production, this is likely to be considered the exception as opposed to the rule. Although product and process development seem quite closely interlinked, history has shown that it is still possible to be extremely successful in business by developing the process technology capability. On many occasions, Japanese companies have been able to take developments from elsewhere in the world and apply their abilities in process technology, allowing them to redefine the market in such sectors as automobiles and consumer electronics.

In certain industries where the coordination of particular manufacturing processes offers potential for improvements in performance, there becomes a need for a different type of technology to support coordination. Where the coordination of two co-located departments can be very difficult, the scope of such technology can be appreciated, and so can the potential benefits. Enterprise Resource Planning (ERP) is an example of a type of indirect process technology which, although working alongside the transformation process, allows better operation-wide understanding that can promote coordination. When it becomes possible to observe, analyse and control operations, the knowledge associated with the process can increase, helping to further direct improvement activities. Indirect process technologies do not necessarily need to be as high tech or as capitally intensive as an ERP system, although what is understood is that they should support and assist, rather than detracting from the direct process so the supporting activities do not become the main focus of operations (Drucker 1955).

Examples of this type of activity are statistical process control (SPC) and the development of management procedures, but could consist of the appliance of science to almost any business activity. SPC uses another type of indirect technology (metrology) to measure business processes to gain a better understanding of the way a process varies. By combining quality, production and process engineering functions, its correct use can develop more consistent and capable processes while developing coordination between functions. The construction of management procedures for certain management activities can help remove or support the judgment of those within the process that can effectively act as a decision support tool 
for management, automating regularly repeated activities. To prevent their maintenance and operations detracting from the primary business process, it is important to view the activities from the perspective of the end user, to assess how the activities contribute to the value that they realise.

Both indirect and direct types of technologies have an important role to play within the operations resource area of operations strategy because they represent the current and future capabilities of a company to perform the transformation process. They also determine the company's ability to continue operating, as they define the rules and systems that maintain control within the operation as well as the specific transformation process. The inclusion of process technology within operations strategy gives specific focus to both the direct and supporting capabilities required in the system to analyse, control and develop operations to achieve the operations strategy. In addition to this, it is the systems that make up a company that have the potential to create, develop or destroy culture, meaning that appreciating the need for consistency when altering them is extremely important. With business processes having direct effects on business culture, there can be a shift away from the view that culture is difficult to define and create, due to the ease with which business processes can be changed (Bossidy and Charan 2002).

\subsubsection{Capacity Strategy}

Out of the different areas of operations resources, capacity planning is probably the area that receives the most attention from traditional practitioners, although the scope of these views is likely to be immediate operations management issues rather than operations strategy. Determining the number of staff that are required to meet the customer demand in the following month to ensure orders can be met is an operational issue rather than a strategic one, although expanding the range of the view makes it strategic. By concentrating on better forecasting or how much inventory should be held, the manager is potentially able to reduce variation in production requirements, which greatly helps improve traditional measures of operating efficiency. However, the effect of this can be to overlook operating issues that are present in the system that may be considerably more costly than the cost of orders that were not met or machines that were not utilised, such as the need to manage large amounts of inventory. Appreciating that a particular system is able to produce certain quantities and respond to certain requirements allows the capacity strategy to be matched to the market's requirements and process's capability.

Through the development of certain approaches to manufacturing, it has been possible to reduce many of the traditional trade-offs, such as low 
inventory with quick response times. Systems such as just-in-time help to control the capacity squeeze that can restrict efficiencies when demand begins to reach capacity. However, what such systems gain in control they potentially lose in flexibility and ultimate efficiency. There still needs to be consideration given to how total demand will change over time. Rather than simply considering how to allocate staff to meet demand, decisions need to be made on the point at which machines need duplicating or replacing to meet demand. This decision can only be made after careful consideration of the long-term market conditions with reference to product and process technologies. Where staffing issues could be thought of as relatively flexible, investing in a new technology not only needs justifying financially, but also has the potential to dramatically alter the market within which it is present.

As stated in the process technology section, the technology available can potentially determine the presence of a market and also define the competitive criteria present in a market. The presence of these two issues relating to the effect of the introduction of a new process technology places additional importance on the decision of whether to invest and, if so, when to invest. The actions of competitors and the market conditions will determine how a company should proceed in its capacity strategy: whether to lead, follow demand, follow the competitor, anticipate or prepare when resources are available. These all have different associated risks and benefits, while also having different effects on the market themselves; as has been said, there are no non-passive actors in an organisation (where the organisation in this case is the whole market) (Callon 1993). The decisions are made considerably more difficult when their results can take a considerable amount of time to realise, such as the construction of a new plant.

Although focusing on the levels of staffing required to meet an order and the introduction of a new process technology in an existing facility can allow demand to be met, there is a time when the squeeze is too great and additional plants are required. The way this situation is addressed can have considerable effects on the organisation's abilities, as there is potential for these decisions to be made on a purely financial basis. In this situation, the case for keeping an old plant is strong, due to the low book value; however, as noted in facilities planning literature, the costs associated with operating can be considerably higher due to many inherent issues (Tompkins et al. 2003). The financial-based argument is also present if the decision has been made to invest in a new plant: to minimise the cost per unit produced, it may be beneficial to build a plant that is able to produce what is required by the market in the most efficient manner possible. With a traditional view of mass production, the way of achieving this is to build a large plant that may have flexibility considered by allowing future expansion. 
Although this approach may again seem the most logical from a financial perspective of being able to produce the parts in the most cost effective manner possible while considering the future, it does not consider a number of additional factors. Although promoting economies of scale through efficient manufacture of parts, when plants increase in size there are also the associated diseconomies of scale, such as communication, hierarchy and bureaucracy overheads. Such a decision also effectively locks the company into a particular investment for a considerable period of time, which is a considerable risk when markets are so changeable. For this reason choosing the smallest size of facility that meets current demand will fulfil both requirements, while leaving future decisions unconstrained. Without such constraints, future bases of operations can be selected to reflect the market needs, rather than available capacity enabling choices that reflect current market conditions. As well as reducing the diseconomies of scale, such an approach could reflect the needs of the customer with reduced lead times, but also offer economies of scope in plants that specialise in particular areas of operations.

The decisions related to capacity need careful consideration with regards to the other areas of operations strategy; however, unlike the other areas, the risks associated with achieving a capacity strategy can be considerably larger. The reason for this is that it is likely to require considerable investment based on current market conditions, process technology and product technology when the decisions are for the future. Unlike the other areas where it may be possible to develop organisational capabilities that are developed in parallel and complement the transformation process, capacity strategy is significantly more tangible. For this reason, an approach to capacity strategy that builds flexibility and promotes the establishment of other capabilities could be considered a way to add intangible aspects to a capacity strategy. Building up capacity through a number of small steps allows for the creation of an organisational network, assisting in developing customer-specific capabilities or promoting the development of focused factories that pursue process excellence across the network. This is effectively how a single company could apply supply network thinking to the way they work to meet their customer demands for capacity, which is the next topic to be discussed.

\subsubsection{Supply Network Strategy}

The transformation process that takes place within a firm can almost never be considered a single activity and is generally made up of a number of components that represent the different elements that make up the product's cost. In defining the value chain, Porter (1985) identified the main 
elements that contribute to part cost and how each part of the business needs to be considered when determining the final selling price. Without considering each particular element, it would not be possible for the part to be supplied, meaning that if an element were missing there would be no selling price.

Figure 1.9 is an example of a value chain for a single company, but it is well documented and understood that a product is not generally created in its entirety within a single firm and passes through a number of stages on its way to the customer. In the same way that is it unlikely that a single company can entirely process and distribute a product, it is also unlikely that the series of companies that produce a product will be arranged sequentially to make a simple chain. Due to end products generally being made up of numerous component parts from different suppliers and being distributed through numerous channels, the companies that work together to supply a product are arranged into networks, as in Figure 1.10. For this reason, the effective management and associated strategy relating to the network will have a considerable effect on a company's ability to supply a product in the required form, while adding value in the most effective way.

Historically, if the strategic processes were carried out on manufacturing

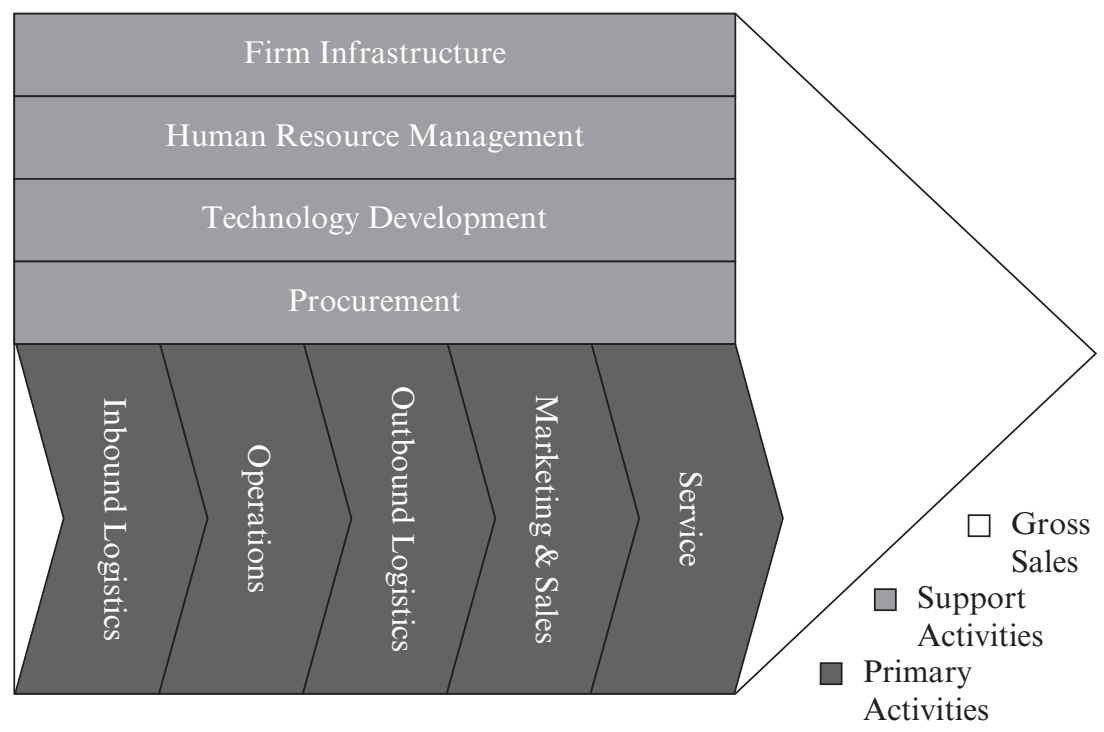

Source: Porter (1985).

Figure 1.9 The value chain 


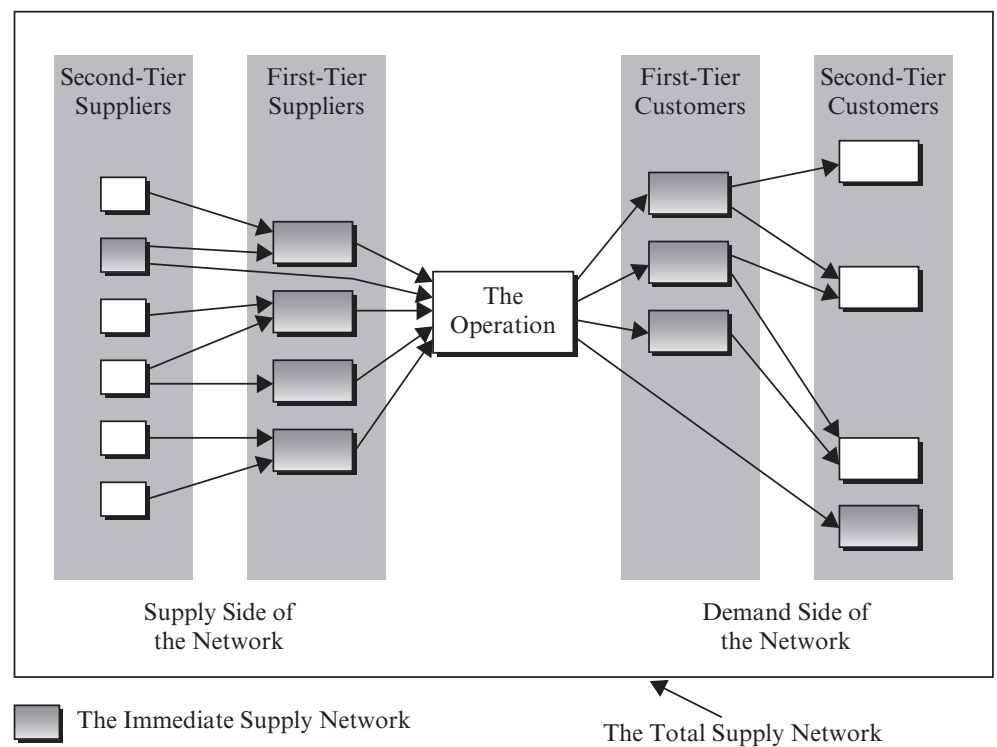

Source: Slack, Chambers and Johnston (2007).

Figure 1.10 A single organisation's supply network

facilities using financially based data, the figures tended to suggest that if supplied parts were made in house, purchasing costs would be reduced and profits could be increased. However, what the financial view did not take into account was that the capabilities required to produce and develop the parts and process also needed considering within the investment argument. Unless all processes were specifically produced for the system, there would also be a difficulty balancing capacity if there was only to be one customer, which may result in less efficient supply or the need to run the suppliers as separate companies, removing some of the benefits of vertical integration. In terms of an organisation, vertical integration is similar in nature to a hierarchically organised company, where the structure slows the movement of information and reduces the company's ability to change. In a vertically integrated company, the problem is considerably more 'plumbed in', with the company only able to supply parts that require the supply chain that is in place. The Raleigh bicycles company and the Ford motor company are both examples of this. Without being able to quickly and effectively alter products and their cost structures in a manner that reflects the needs of the market, there may be significant repercussions for the health of the business. 
The developments in the east of networks of supplying companies working together demonstrated that vertical integration benefits were possible without the associated restrictions. With the breaking down of traditional supplier-customer barriers, combined with developing abilities to supply effectively through supplier development activities, this could greatly increase the ease with which two companies could work together. When it became possible to realistically choose between internally and externally made parts, both with consistent quality and supplier performance, the choices for purchasers increased, increasing the significance of developing a suitable supply network. With a single company being both supplier and customer to many firms, the hierarchy is reduced and the effect of a single company's actions on other companies within the network is also reduced. Companies with a significant number of supply links or spokes are able to reduce the effect of disturbances within the network, reducing network shock (which is caused when a company within a network stops functioning). Such a network can also allow companies to more effectively tailor their supplier network to take account of the requirements of a market at a particular time.

Flexibility and security are not the only benefits of working effective within a supply network: making the choice of whether to make or buy allows companies to focus their operations on specific capabilities that define their operations. When a company has the choice of what it makes, it is able to make a choice that reflects the needs of its customer but also one that is in line with what the company considers important. The company no longer has to produce everything it needs or only outsource commodity products, but can outsource products that may not fit with its specific development strategy or core competences (Hamel and Prahalad 1990). This increases a company's ability to specialise and excel in particular areas, by developing capabilities it considers to be particularly critical to adding value for its customer. This can also allow organisations to source particular elements of the products that are able to benefit from low-cost labour or particular capabilities that may not be present locally.

Presence within a network allows further benefits; rather than simply outsourcing the supply of pre-designed parts, there is potential for greater levels of collaboration within networks. Enabled by the use of indirect process technologies, geographically disparate collaborations can be coordinated, allowing complex new products to be developed using knowledge present across a network that prevents a single organisation being responsible for the associated financial risk. Taking this idea further, virtual enterprises are possible that can be initiated by a single entrepreneur's idea, with all elements being outsourced. Such 
approaches being so heavily reliant on distributing risk and establishing trust, that may not have been present within traditionally run organisations, increases the importance of developing an appropriate supply network strategy. Television programmes and Hollywood films are both examples of how complex activities can be undertaken outside a traditional organisational structure. These activities do show the need for additional elements that require consideration to facilitate activities, such as personal networks and latent organisation, that are alternate structures to base activities upon (Robins 1993; Starkey, Barnatt and Tempest 2000).

\subsection{PROCESS OF OPERATIONS STRATEGY}

As outlined above, there is a great need to consider many areas of business to be able to create an appropriate operations strategy. Even specific areas have numerous sub-topics that need taking into account, specifically operations resources. Focusing on any of the areas has both the potential to create an effective strategy and improved business results, while simultaneously leading an organisation in the wrong direction. In this introduction, it has been the aim to illustrate how in all areas of operations strategy it is not only possible but important to consider the other elements; however, there is still the problem of how to reconcile market requirements with operations resources. Rather than solve this issue, it has been thought that focusing on the improvement of operations may be a substitute for a carefully formulated operations strategy. This is potentially why companies choose prescriptive approaches to operations improvement, such as Six Sigma or TQM, as they consider both market requirements and the development of operations resources. Although such approaches may consider the necessary elements, it is unlikely they will be suitable unless alterations are made to meet the needs of a specific business's requirements.

Specific situations require that there is a degree of 'fit' between the market requirements and the operations capability, which the more prescriptive approaches may not take into account. GE demonstrates that to be successful, it may be necessary to exert pressure on a system through incentives and authority to make a company change so that it fits a particular operations management approach. Unfortunately, this can be expensive and time consuming, meaning the need to develop an appropriate operations strategy becomes important, when companies may not have the necessary financial reserves or time requirements. A strategy should be formulated that reflects both the needs of the market and 
operations capability, otherwise additional attention needs to be focused on developing the market or the operations so that a particular strategy can be achieved. This is likely to include allowances for time, effort and investment to understand how to move from the content to create an appropriate strategy that reflects a company's needs. The operations strategy process uses the elements of content with market requirement data to develop the strategy, that systematically account for the requirements of the system. The result is that the strategy reflects the needs of the organisation to enable introduction, to reduce time and cost but simultaneously improve the results of the activity. Approaches to operations strategy process will be discussed in the next chapter.

\subsection{SUMMARY}

The aim of this chapter has been to introduce the reader to the subject of strategy and, in particular, operations strategy. The theme throughout has been to stress the importance of each aspect, and the reasons for considering multiple areas in the development of a strategy but also how to use it within an organisation. In the global business arena, 'business as usual' no longer exists as the world is constantly changing and competing is becoming more difficult. Companies can no longer formulate general strategies that result in slight changes in performance; investments need to be directed to add maximum value to the operations. This means an operations strategy should be formulated that is specific to the needs of the organisation, to allow for the directed improvements the market requires while also building capabilities that define the company against its competitors. Capability development has also been a theme of the introduction that reflects the need for a strategic capability to be able to combine and consider numerous elements of strategic information to convert strategic ideas into organisational activities.

The capabilities that have been mentioned, that are necessary and are developed within the specific areas of operations strategy, represent intangible strategic resources that are not necessarily dependent on the details of a specific strategy. Through the development of capabilities that assist in strategy realisation, a company could develop a sustainable competitive advantage, being able to apply these abilities to the most appropriate strategy. It is the presence of specific company-specific capabilities that allow the coordination that is required for the implementation of a strategy that is often considered more important than formulation. Without the implementation of a strategy, there will be no change in a company's operations, meaning the time and effort spent on the process represent 
a very poor use of resources. Even with substantial resources and good strategic fit, the implementation process of a new strategy can still be a very complex process. For this reason, the importance of having a good understanding of the content and process of operations strategy is no less than having good skills in the implementation of strategy. 